\title{
SINGLE-VALUED REPRESENTATION OF SET-VALUED MAPPINGS
}

BY

A. D. IOFFE

\begin{abstract}
It is shown that the graph of a set-valued mapping satisfying typical conditions which guarantee the existence of measurable selections can be represented as the union of graphs of measurable single-valued mappings depending continuously on a parameter running through some Polish space.
\end{abstract}

1. Introduction. The principal results of this paper show that the graphs of certain set-valued mappings are precisely the ranges of Carathéodory-type functions. To be more specific, let a set-valued mapping $M$ from a set $T$ into another set $X$ (we abbreviate $M: T \rightarrow X$ ) be given. Let $Z$ be another set, and $f: T \times Z \rightarrow X$ a single-valued mapping. We shall say that the pair $(Z, f)$ represents $M$ if $f(t, Z)=M(t)$ whenever $M(t) \neq \varnothing$. Here as usual,

$$
f(t, Z)=\{x \in X \mid x=f(t, z) \text { for some } z \in Z\} \text {. }
$$

In what follows, we impose certain restrictions on the class of $T, X$ and $M$ to be considered. As usual, $T$ is endowed with a $\sigma$-algebra $\mathfrak{M}$ of subsets so that $(T, \mathfrak{M})$ is a measurable space, and $X$ is a topological space, usually Polish. If $X$ and $Z$ are topological spaces and $(T, \mathfrak{M})$ is a measurable space, then the mapping $f: T \times Z \rightarrow X$ will be called $\mathfrak{M}$-Carathéodory if $f(t, \cdot)$ is continuous for any $t \in T$ and $f(\cdot, z)$ is $\mathfrak{M}$-measurable for any $z \in Z$. We shall say that a set-valued mapping $M: T \rightarrow X$ is $\mathfrak{M}$-analytic if $\operatorname{dom} M=$ $\{t \mid M(t) \neq \varnothing\}$ (the domain of $M$ ) belongs to $\mathfrak{M}$ and there are a Polish space $Z$ and an $\mathfrak{M}$-Carathéodory mapping $f: T \times Z \rightarrow X$ such that the pair $(Z, f)$ represents $M$.

Using this terminology, we can formulate the purpose of the paper as to describe the class of analytic set-valued mappings. It turns out that this class includes most of the set-valued mappings for which measurable selection theorems hold, such as those of Aumann [1], Kuratowski and Ryll-Nardzewski [8], Leese [9], Novikov [12], Rokhlin [13] and certain others. Moreover, all these theorems are immediate and trivial corollaries of the corresponding representation theorems being proved here. (Indeed, if $M$ is $\mathfrak{M}$-analytic and is represented by $(Z, f)$, then, an arbitrary $z \in Z$ having been fixed, the mapping $t \rightarrow f(t, z)$ is a selector of $M$.)

Received by the editors November 7, 1977.

AMS (MOS) subject classifications (1970). Primary 28A05, 54H05. 
Thus our theorems cover the class of closed-valued mappings (Theorem 1) and set-valued mappings with Suslin graphs (Theorem 5). In the case when $\mathfrak{M}$ is stable under the $A$-operation of Suslin, the latter class simply coincides with the class of $\mathfrak{M}$-analytic set-valued mappings (Corollary 5.4). Other results stated as corollaries include a refinement of the Novikov-Castaing representation by countable system of measurable selections (Corollary 1.3), a representation theorem for convex-valued mappings by linear operators depending measurably on $t$ (Corollary 1.4) and certain other facts. We hope to publish elsewhere a more thorough study of convex-valued mappings and mappings with uncountable graphs.

The word multifunction will be used in the same sense as set-valued mapping. The set

$$
\operatorname{Gr} M=\{(t, x) \in T \times X \mid x \in M(t)\}
$$

is the graph of $M$. Other notions and notations will be explained in appropriate places.

The results presented here were partly announced in [6].

I wish to thank the referee for helpful comments.

2. Closed-valued multifunctions. If $X$ is a topological space, then $\mathscr{U}(X)$ and $\mathscr{F}(X)$, or merely $\mathcal{Q}$ and $\mathscr{F}$, will be the families of all open and closed subsets of $X$, respectively. If $\mathcal{C}$ is a family of subsets of $T$, then $\Sigma(\mathcal{C})$ will be the minimal $\sigma$-algebra containing $\mathcal{C}$. If $A \subset X$, then

$$
\begin{aligned}
& M^{-}(A)=\{t \in T \mid M(t) \cap A \neq \varnothing\}, \\
& M^{-}(\mathcal{Q})=\left\{E \subset T \mid E=M^{-}(U) \text { for some } U \in \mathcal{U}(X)\right\},
\end{aligned}
$$
and so on.

In this section we shall consider closed-valued multifunctions from $T$ into $X$, that is, those whose values are closed subsets of $T$.

Theorem 1. Let $X$ be a Polish (resp. compact metrizable) space, and let $M$ be a set-valued mapping from $T$ into $X$ such that all sets $M(t)$ are nonempty and closed. Let $\mathfrak{M}=\Sigma\left(M^{-}(\mathcal{Q})\right)$. Then $M$ is $\mathfrak{M}$-analytic.

Moreover, if $\rho$ is a metric on $X$ compatible with the topological structure, then among the pairs $(Z, f)$ representing $M$, there is one such that $Z$ is a complete metric (resp. compact metric) space, $f$ is $\mathfrak{M}$-Carathéodory and

$$
\rho(f(t, z), f(t, w)) \leqslant(1+\rho(f(t, z), f(t, w))) r(z, w)
$$

for all $z, w \in Z, t \in T$, where $r(\cdot, \cdot)$ is the distance in $Z$.

The proof of the theorem will be given in the next section.

If $(T, \mathfrak{M})$ is a measurable space, then the set-valued mapping $M$ is called $\mathfrak{M}$-measurable if $\boldsymbol{M}^{-}(\mathcal{U}) \subset \mathfrak{M}$. 
Corollary 1.1. Let $(T, \mathfrak{M})$ be a measurable space, and let $M$ be a measurable closed-valued multifunction from $T$ into a Polish (resp. compact metrizable) space $X$. Then the conclusion of the theorem holds.

Proof. This is obvious.

Theorem 1 was originally proved in the form of this corollary [6], but using $\sigma$-algebras more closely connected with $M$ might be more natural (cf. Leese [10], Levin [11]). The corollary also shows that, assuming dom $M$ equal to $T$, we lose no generality. To simplify argumentation, we shall frequently use this assumption.

Corollary 1.2. Let $T$ be a Polish space, and let $X$ be a compact metrizable space (resp. a Polish space which is the union of countably many compact metrizable spaces). Let $M$ be a closed-valued multifunction from $T$ into $X$ such that $\operatorname{dom} M=T$ and $\operatorname{Gr} M$ is a Borel subset of $T \times X$. Then there are $a$ compact metrizable (resp. a Polish) space $Z$ and a Borel function $f: T \times Z \rightarrow X$ satisfying (1) (under arbitrary choice of $\rho$ and suitable choice of the distance $r$ in $Z)$, and such that the pair $(Z, r)$ represents $M$.

Proof. By Novikov's projection theorem [12], $M^{-}(F)$ is Borel for any $F \in \mathscr{F}$. Hence $M^{-}(U)$ is Borel for any $U \in \mathcal{U}$ (since $\mathcal{U} \subset \mathscr{F}_{0}$ ). Choose $Z$ and $f$ in accordance with Theorem 1 . Then $f(\cdot, z)$ is a Borel function for any $z \in Z$ and $f(t, \cdot)$ is a continuous function for any $t \in T$ (due to (1)). Hence $f$ itself is a Borel function.

Let us fix a metric $\rho$ in $X$ such that diam $X<\infty$. Then the space $B(T, X)$ of all mappings from $T$ into $X$ with the metric defined by

$$
d(x(\cdot), y(\cdot))=\sup _{t \in T} \rho(x(t), y(t))
$$

is a complete metric space. To any set $Q \subset B(T, X)$ corresponds a set-valued mapping $M_{Q}: T \rightarrow X$ defined by

$$
M_{Q}(t)=\{x \in X \mid x=x(t) \text { for some } x(\cdot) \in Q\} .
$$

Note also that the closure in $B(T, X)$ of any set consisting of $\mathfrak{M}$-measurable mappings also consists of $\mathfrak{M}$-measurable mappings since measurability is preserved under uniform convergence.

COROLlARY 1.3. Let $M$ and $\mathfrak{M}$ be the same as in the theorem. Then there is a countable family $Q$ of $\mathfrak{M}$-measurable mappings from $T$ into $X$ such that

$$
M(t)=M_{\bar{Q}}(t), \quad \forall t \in T,
$$

where $\bar{Q}$ is the closure of $Q$ in $B(T, X)$. Moreover, if $X$ is a compact metric space, then $Q$ can be chosen to be precompact in $B(T, X)$.

Proof. Take $Z$ and $f$ as in Theorem 1 and consider the mapping $z \rightarrow f(\cdot, z)$ from $Z$ into $B(T, X)$. According to (1), this mapping is continuous. Hence it 
suffices to choose a dense countable subset $\left\{z_{1}, z_{2}, \ldots\right\} \subset Z$ and set

$$
Q=\left\{f\left(\cdot, z_{i}\right) \mid i=1,2, \ldots\right\} .
$$

This corollary gives a refined version of the Novikov-Castaing representation of a closed-valued multifunction by a countable family of measurable selections [3], [12]. According to them, there is a countable family $\left\{x_{1}(\cdot), x_{2}(\cdot), \ldots\right\}$ of measurable selections of $M$ such that for any $t$ the set $M(t)$ is the closure of the set $\left\{x \in X \mid x=x_{i}(t)\right.$ for some $\left.i=1,2, \ldots\right\}$. Clearly, any $Q$ in Corollary 1.3 gives such a representation but not vice versa.

We recall, in connection with Novikov-Castaing representations, that, for closed-valued multifunctions, the existence of such a representation by a family of $\mathfrak{M}$-measurable selections is equivalent to $\mathfrak{M}$-measurability of the set-valued mapping in question.

COROllary 1.4. Let $X$ be a separable Banach space, and let $M$ be a multifunction from $T$ into $X$ with nonempty closed and convex values. Then there are another separable Banach space $Y$, a closed convex set $Z \subset Y$ and $a$ $\mathfrak{M}$-Carathéodory mapping $f: T \times Y \rightarrow X$ which is affine and nonexpansive in the second argument and such that the pair $(Z, f)$ represents the multifunction M.

Proof. Let $a(t)$ be an arbitrary $\mathfrak{M}$-measurable selection of $M$. Denote by $B_{n}$ the ball of radius $n$ about the origin in $X$, and consider the multifunctions

$$
P(t)=M(t)-a(t), \quad P_{n}(t)=P(t) \cap B_{n} .
$$

All sets $P(t), P_{n}(t)$ are obviously nonempty closed and convex. Also clear is that the set-valued mappings $P$ and $P_{n}$ are $\mathfrak{M}$-measurable. Indeed, if $x_{k}(\cdot)$, $k=1,2, \ldots$, is a family of $\mathfrak{M}$-measurable selections of $M$ giving a Novikov-Castaing representation for $M$, then $u_{k}(t)=x_{k}(t)-a(t)$ and $w_{k}(t)=$ $\left(\max \left(1,\left\|u_{k}(t)\right\| / n\right)\right)^{-1} u_{k}(t)$ are also $\mathfrak{M}$-measurable and the families $\left\{u_{k}(\cdot) \mid k\right.$ $=1,2, \ldots\}$ and $\left\{w_{k}(\cdot) \mid k=1,2, \ldots\right\}$ are Novikov-Castaing representations for $P$ and $P_{n}$, respectively.

We can consider every $P_{n}$ as a set-valued mapping from $T$ into $B_{n}$, the latter endowed with the metric induced by the norm of $X$. Let $Q_{n}$ be a countable family of measurable selections of $P_{n}$ chosen in accordance with Corollary 1.3. Then $Q=\cup Q_{n}$ consists of bounded $\mathfrak{M}$-measurable mappings from $T$ into $X$. Let $Y$ be the completion of the linear hull of $Q$ with respect to the norm $\|y(\cdot)\|_{C}=\sup _{t}\|y(t)\|_{X}$. Then $Y$ is a separable Banach space. Let $Z$ be the closure in $Y$ of the convex hull of $Q$. Then $z(\cdot) \in Z$ implies that $z(t) \in P(t)$ for all $t$ since each $P(t)$ is convex and closed. Moreover, inasmuch as $P_{n}(t)=M_{\bar{Q}_{n}}(t)$, we have that for any $t \in T$ and any $x \in P(t)$, there is a $z(\cdot) \in Z$ such that $x=z(t)$.

Consider finally, for any $t$, the linear operator $A(t): Y \rightarrow X$ defined by 
$A(t) y(\cdot)=y(t)$. According to this definition, the mapping $t \rightarrow A(t) y(\cdot)$ is $\mathfrak{M}$-measurable for any $y(\cdot) \in Y$ and $\|A(t)\|<1$ for any $t \in T$. It remains to set $f(t, y(\cdot))=A(t) y(\cdot) \rightarrow a(t)$.

REMARK. In [6] (the last statement of Theorem 1) the latter result was wrongly announced for arbitrary Fréchet spaces, not necessarily Banach. As has been pointed out by the referee, this will hold under the additional assumption that the set-valued mapping $M$ assumes values in a fixed bounded set.

3. Proof of Theorem 1. In what follows, $N=\{1,2, \ldots\}$ and $\Re=N^{N}$ is the Baire space of sequences of integers $i=\left(i_{1}, i_{2}, \ldots\right)$ with the metric defined by

$$
b(i, j)=\sum_{k=1}^{\infty} 2^{-k} \frac{\left|i_{k}-j_{k}\right|}{1+\left|i_{k}-j_{k}\right|} .
$$

If $i=\left(i_{1}, i_{2}, \ldots\right)$ and $n>1$, then $i \mid n=\left(i_{1}, \ldots, i_{n}\right)$. The same symbol, $i \mid n$, will be also used to denote $n$-tuples of integers with no reference to any element of $\Re$. Given $i \mid n$, the set

$$
\Re_{i \mid n}=\{j \in \Re|j| n=i \mid n\}
$$

is called a Baire interval of rank $n$. Baire intervals are both open and closed in $\pi$.

Let $X$ be a Polish space. Throughout the section we fix a metric $\rho$ in $X$ which turns $X$ into a complete metric space.

We shall construct the desired space $Z$ as a closed subset of the Baire space $\Re$ which will be compact if $X$ is compact. The principal part of the proof consists of choosing a family $\mathfrak{T}$ of set-valued mappings from $T$ into $X$ which has the following properties:

(i) $M \in \mathfrak{N}$;

(ii) if $R \in \mathfrak{N}$, then $\operatorname{dom} R=T$;

(iii) $R^{-}$(थ) $\subset \Sigma\left(M^{-}(\right.$U) ) whenever $R \in \Re$;

(iv) to any $i \mid n$ such that $Z \cap \Re_{i \mid n} \neq \varnothing$ corresponds a set-valued mapping $M_{i \mid n} \in \mathfrak{N}$ such that

$$
\operatorname{diam} M_{i \mid n}(t)<2^{-n}, \quad \forall t \in T
$$

(v) for all $t \in T$ and $i \mid n$,

$$
\bigcup_{j \in Z_{i \mid(n-1)}} M_{j \mid n}(t)=M_{i \mid(n-1)}(t)
$$

Here we have denoted

$$
\begin{gathered}
Z_{i \mid n}=Z \cap \Re_{i \mid n}, \\
\Re_{i \mid 0}=\Re, \quad M_{i \mid 0}=M \text { for all } i \in Z .
\end{gathered}
$$


Such a family having been built, the theorem is proved as follows. Let us set

$$
f(t, i)=\bigcap_{n>1} \bar{M}_{i \mid n}(t),
$$

where the bar over $M_{i \mid n}(t)$ denotes the closure of $M_{i \mid n}(t)$. We have $\bar{M}_{i \mid n} \neq \varnothing$ (by (ii)), diam $\bar{M}_{i \mid n}(t)<2^{-n}$ (by (iv)) and $\bar{M}_{i \mid(n+1)}(t) \subset \bar{M}_{i \mid n}(t)$ (by (v)). Hence the intersection in (2) contains exactly one element and $f$ is actually a single-valued mapping from $T \times Z$ into $X$. It follows also from (v) that $f(t, i) \in M(t)$. Hence $f(t, Z) \subset M(t)$.

Let us show that, in fact, $f(t, Z)=M(t)$ or, in other words, that for any given $t \in T, x \in M(t)$, there is an $i \in Z$ such that $f(t, i)=x$. Applying (v) to $n=1$, we find $i^{1} \in Z$ such that $x \in M_{i^{1} \mid 1}(t)$. Applying (v) again to $n=2$, $i=i^{1}$, we find $i^{2} \in Z$ such that $i^{2}\left|1=i^{1}\right| 1$ and $x \in M_{i^{2} \mid 2}(t)$. Going on with this procedure, we find a sequence $\left\{i^{1}, i^{2}, \ldots\right\}$ of elements of $Z$ such that $i^{k}\left|k=i^{k+1}\right| k$ and $x \in M_{i^{k} \mid k}(t)$ for all $k$. The first of these relations shows that $b\left(i^{k}, i^{k+1}\right)<2^{-(k-1)}$ so that the sequence $\left\{i^{1}, i^{2}, \ldots\right\}$ converges in $\Re$ to a certain $i$. Since $Z$ is closed, $i \in Z$. On the other hand, it follows that $i^{k}|k=i| k$ for any $k$, hence $x \in M_{i \mid k}(t)$ for all $k$ and $f(t, i)=x$.

Thus the pair $(Z, f)$ actually represents $M$ and it remains to verify that $f$ is $\mathfrak{M}$-measurable in $t$ and satisfies (1).

To check measurability, it suffices to demonstrate that $\{t \mid f(t, i) \in F\} \in \mathfrak{M}$ whenever $F \in \mathscr{F}, i \in Z$. This follows from the obvious equality

$$
\{t \mid f(t, i) \in F\}=\bigcap_{\substack{\varepsilon>0 \\ n>1}} M_{i \mid n}^{-}\left(F_{\varepsilon}\right), \quad F_{\varepsilon}=\{x \mid \rho(x, F)<\varepsilon\},
$$

which in turn is a direct consequence of definition (2).

It remains to prove that there is another metric $r$ on $Z$ such that

$$
\rho(f(t, i), f(t, j)) \leqslant(1+\rho(f(t, i), f(t, j))) r(i, j)
$$

for all $i, j \in Z, t \in T$. Take $i, j \in Z$. If $b(i, j)>1 / 8$, then

$$
\begin{aligned}
\rho(f(t, i), f(t, j)) & \leqslant 1+\rho(f(t, i), f(t, j)) \\
& \leqslant 8(1+\rho(f(t, i), f(t, j))) b(i, j) .
\end{aligned}
$$

If $b(i, j)<1 / 8$, then there is $n>1$ such that $2^{-(n+3)}<b(i, j)<2^{-(n+2)}$. Because of the right-hand side of this inequality, $i|n=j| n$, hence

$$
f(t, i) \in \bar{M}_{i \mid n}(t), \quad f(t, j) \in \bar{M}_{i \mid n}(t)
$$

and, according to (iv),

$$
\begin{aligned}
\rho(f(t, i), f(t, j)) & \leqslant 2^{-n}<8 b(i, j) \\
& \leqslant 8(1+\rho(f(t, i), f(t, j))) b(i, j) .
\end{aligned}
$$

As follows from (4) and (5), inequality (3) holds with $r(i, j)=8 b(i, j)$. 
Thus we have to produce a set $Z$ and a family $\mathscr{T}$ satisfying the above listed properties (i)-(v).

Let $\left\{U_{1}^{m}, U_{2}^{m}, \ldots\right\}$ be a covering of $X$ by open balls with radius $<2^{-m}$, and let $c_{m}$ be the number of balls in the covering (which may be equal to $+\infty)$. If $X$ is compact, we shall take coverings with $c_{m}<\infty$. If $X$ is not compact, $c_{m}=\infty$ beginning with a certain $m_{0}$.

Let

$$
Z=\left\{i \in \Re \mid i_{k}<c_{k}+1, k=1,2, \ldots\right\} .
$$

In other words, $i_{k}<c_{k}$ if $c_{k}<\infty$ and $i_{k}$ may be an arbitrary integer if $c_{k}=\infty$. The set-valued mappings $M_{i \mid n}$ will be defined recursively.

Let $M_{i \mid 0}=M$ for all $i \in Z$ and suppose we have already found multifunctions $M_{i \mid n}$ satisfying (ii)-(v) for all $i \in Z$ and $n<m$. The multifunction $M_{i \mid(m+1)}$ will be then defined as follows. Take $i \in Z$, and let $i_{m+1}=s$. Let

$$
T_{k}=M_{i \mid m}^{-}\left(U_{k}^{m+1}\right), \quad k=1,2, \ldots,
$$

and

$$
M_{i \mid(m+1)}(t)= \begin{cases}M_{i \mid m}(t) \cap U_{s}^{m+1}, & \text { if } t \in T_{s}, \\ M_{i \mid m}(t) \cap U_{s+1}^{m+1}, & \text { if } t \in T_{s+1} \backslash T_{s}, \\ \cdots & \text { if } t \in T_{1} \backslash \cup \cup_{k=s}^{c_{m+1}} T_{k}, \\ M_{i \mid m}(t) \cap U_{1}^{m+1}, & \\ M_{i \mid m}(t) \cap U_{s-1}^{m+1}, & \text { if } t \in T_{s-1} \backslash \cup_{k \neq s-1} T_{k} .\end{cases}
$$

Since $\operatorname{dom} M_{i \mid n}=T$ and $\cup_{k} U_{k}^{m+1}=X$, we have $\operatorname{dom} M_{i \mid(m+1)}=T$ so that (ii) holds for $M_{i \mid(m+1)}$. If $U \subset X$ is open, then

$$
\begin{aligned}
M_{i \mid(m+1)}^{-}(U)= & \left(M_{i \mid m}^{-}\left(U \cap U_{s}^{m+1}\right) \cap T_{s}\right) \\
& \cup\left(M_{i \mid m}^{-}\left(U \cap U_{s+1}^{m+1}\right) \cap\left(T_{s+1} \backslash T_{s}\right)\right) \cup \ldots \\
& \cup\left(M^{-}\left(U \cap U_{s-1}^{m+1}\right) \cap\left(T_{s-1} \backslash \cup_{k \neq s-1} T_{k}\right)\right) .
\end{aligned}
$$

Since $M_{i \mid m}(V) \in \mathfrak{M}$ for any open $V$, this equality shows that $M_{i \mid(m+1)} \in \mathfrak{M}$. Thus (iii) holds for $R=M_{i \mid(m+1)}$. The validity of (iv) follows from the definition of $M_{i \mid(m+1)}$ :

$$
\operatorname{diam} M_{i \mid(m+1)}(t)<\sup _{k} \operatorname{diam} U_{k}^{m+1}<2^{-(m+1)} .
$$

And finally, (v) results from the already used fact that $\cup_{k} U_{k}^{m+1}=X$.

This completes the proof of the theorem.

4. General description of analytic multifunctions. To give the characterization of analytic set-valued mappings, we need some known facts concerning the $A$-operation of Suslin (see for instance [4], [5], [14]). Recall the definition. 
Let $C_{i \mid n}$ be a collection of sets ( $i \mid n$ ranging the whole set of finite sequences of positive integers). The Suslin operation assigns to each such collection the set

$$
C=\bigcup_{i \in \Re} \bigcap_{n} C_{i \mid n}
$$

If $\mathcal{C}$ is a family of subsets of $T$ and every $C_{i \mid n}$ belongs to $\mathcal{C}$, then $C$ is called $\mathcal{C}$-Suslin. The family of all $\mathcal{C}$-Suslin sets will be denoted by $\mathbb{Q}(\mathcal{C})$.

Proposition 2. $\mathbb{Q}(\mathbb{Q}(\mathcal{C}))=\mathbb{Q}(\mathcal{C})$.

Proof. See for example [2], [14], [15].

Other definitions and notations to be used here are the following. Let $\mathcal{E}$ be a family of subsets of another set $W$; then

$$
\mathcal{C} \times \mathcal{E}=\{C \times E \subset T \times W \mid C \in \mathcal{C}, E \in \mathcal{E}\}
$$

If $X$ is a topological space, then $\mathscr{B}(X)$ will denote the $\sigma$-algebra of Borel subsets of $X$ (the one generated by $\mathscr{F}(X)$ ). If $\mathfrak{M}_{1}$ and $\mathfrak{M}_{2}$ are $\sigma$-algebras, then $\mathfrak{M}_{1} \otimes \mathfrak{M}_{2}=\Sigma\left(\mathfrak{M}_{1} \otimes \mathfrak{M}_{2}\right)$ is the product algebra. Recall that in a Polish space, $\mathscr{B}(X)$ coincides with the minimal family containing $\mathscr{F}(X)$ and countable unions and intersections of its elements.

COROllary 2.1. Let $\mathcal{C}$ be a family of subsets of $T$, and let $\mathscr{B}$ be the minimal family containing $C$ countable unions and intersections. Then

$$
\mathbb{Q}(\mathscr{B})=\mathscr{Q}(\mathcal{C})
$$

In particular, if $X$ is a Polish space, then

$$
\mathscr{Q}(\mathscr{F})=\mathbb{Q}(\mathscr{B}(X)) \text {. }
$$

Proof. This follows from the fact that countable union and intersection are particular cases of the $A$-operation.

Corollary 2.2. Let $\mathfrak{M}$ be a $\sigma$-algebra and $X$ a Polish space. Then

$$
\mathfrak{Q}(\mathfrak{R} \times \mathscr{F})=\mathscr{Q}(\mathfrak{M} \otimes \mathfrak{B}(X)) \text {. }
$$

Proposition 3. Let $\mathcal{C}$ be a family of subsets of $T$. Then any $C \in \mathbb{Q}(\mathcal{C})$ is the domain of a closed-valued multifunction from $T$ into $\Re$ whose graph belongs to $(\mathcal{C} \times \mathcal{F}(\mathcal{T}))_{o \delta}$.

Conversely, if $X$ is a Polish space, $\varnothing \in \mathcal{C}$ and $Q \in \mathbb{Q}(\mathcal{C} \times \mathscr{B}(X))$, then $\operatorname{pr}_{T} Q$, the projection of $Q$ on $T$, belongs to $\mathcal{Q}(\mathcal{C})$.

PROof. The proof of the second part can be found, say, in [5] and [14]. In fact, the proof of the first part is also contained there but in neither of the references is the closedness property emphasized. Therefore we shall describe the construction.

Let

$$
C=\bigcup_{i \in \Re} \bigcap_{n} C_{i \mid n}
$$


and let

$$
Q=\left\{(t, i) \in T \times \Re \mid t \in \bigcap_{n} C_{i \mid n}\right\}=\bigcup_{i \in \Re}\left(\bigcap_{n} C_{i \mid n}\right) \times\{i\} .
$$

Clearly, $\operatorname{pr}_{T} Q=C$. Consider the multifunction $t \rightarrow Q(t)=\{i \in \Re \mid(t, i) \in$ $Q$ \} whose graph is $Q$. Let us verify that each $Q(t)$ is closed. If $i^{k} \rightarrow i$ when $k \rightarrow \infty$, then for any fixed $n, i^{k}|n=i| n$ beginning with a certain $k(n)$. If $i^{k} \in Q(t)$ for all $k$, then $t \in C_{i^{k} \mid n}$ for all $k$ and $n$, hence $t \in C_{i \mid n}$ for all $n$ and $i \in Q(t)$.

The proof that $Q$ belongs to $(\mathcal{C} \times \mathscr{F}(\mathscr{T}))_{o \delta}$ is straightforward and based on the fact that the sets $Q_{i \mid n}=\left(\cap_{n} C_{i \mid n}\right) \times\{i\}$ corresponding to different $i \mid n$ are disjoint, which allows one to change the places of the signs of union and intersection in the above equality for $Q$.

Now we are ready to prove the main results of this section.

Theorem 4. Let $\mathfrak{M}$ be a $\sigma$-algebra of subsets of $T$, and let $X$ be a Polish space. Then the graph of any $M$-analytic multifunction from $T$ into $X$ belongs to $\mathscr{Q}(\mathfrak{M} \otimes \mathfrak{B}(X))$.

Proof. If $M$ is $\mathfrak{M}$-analytic, then $\operatorname{dom} M \in \mathfrak{M}$ and there are a Polish space $Z$ and an $\mathfrak{M}$-Carathéodory mapping $f: T \times Z \rightarrow X$ such that $(Z, f)$ represents $M$. Since any Polish space is a continuous image of $\mathscr{T}$, there is no loss of generality if we assume that $Z=\Re$.

Let us set

$$
M_{i \mid n}(t)= \begin{cases}\overline{f\left(t, \mathscr{\Re}_{i \mid n}\right)}, & \text { if } t \in \operatorname{dom} M, \\ \varnothing, & \text { if } t \notin \operatorname{dom} M\end{cases}
$$

(the bar denotes closure). By definition, the multifunction $M_{i \mid n}$ is closed-valued and $\operatorname{dom} M_{i \mid n}=\operatorname{dom} M \in \mathfrak{M}$. It is easy to see that $\operatorname{Gr} M_{i \mid n} \in \mathfrak{M} \otimes$ $\mathscr{B}(X)$. (Indeed, if $\left\{i^{1}, i^{2}, \ldots\right\}$ is a dense countable set in $\Re_{i \mid n}$, then the sequence $\left\{f\left(t, i^{1}\right), f\left(t, i^{2}\right), \ldots\right\}$ is a Novikov-Castaing representation for $M_{i \mid n}$ by $\mathfrak{M}$-measurable selections. Since $M_{i \mid n}$ is closed-valued, the inclusion $\operatorname{Gr} M_{i \mid n}$ $\in \mathfrak{M} \otimes \mathfrak{B}(X)$ follows.)

We have, furthermore,

$$
\bigcap_{n=1}^{\infty} \overline{f\left(t, \Re_{i \mid n}\right)}=\bigcap_{n=1}^{\infty} f\left(t, \Re_{i \mid n}\right)=\{f(t, i)\}
$$

(because diam $\mathcal{T}_{i \mid n} \rightarrow 0$ and $f(t, \cdot)$ is continuous) whence

$$
M(t)=\bigcup_{i \in \Re}\{f(t, i)\}=\bigcup_{i \in \Re} \bigcap_{n} M_{i \mid n}(t) .
$$

This is the same as

$$
\text { Gr } M=\bigcup_{i \in \Re} \bigcap_{n} \operatorname{Gr} M_{i \mid n} \text {. }
$$


Theorem 5. Let $\mathcal{C}$ be a family of subsets of $T$ containing $\varnothing$, and let $X$ be $a$ Polish space. Let $M$ be a set-valued mapping from $T$ into $X$ with $\mathrm{Gr} M \in \mathbb{Q}(\mathcal{C}$ $\times \mathfrak{B}(X))$. Denote $\mathfrak{M}=\Sigma(\mathbb{Q}(\mathcal{C}))$. Then $M$ is $\mathfrak{M}$-analytic.

Proof. By virtue of Corollary 2.1,

$$
\text { Gr } M=\bigcup_{i \in \mathscr{N}} \bigcap_{n}\left(C_{i \mid n} \times F_{i \mid n}\right),
$$

where $C_{i \mid n} \in \mathcal{C}$ and $F_{i \mid n} \in \mathcal{F}(X)$. By Proposition 3,

$$
\text { Gr } M=\operatorname{pr}_{T \times X} Q,
$$

where

$$
Q=\left\{(t, x, i) \in T \times X \times \Re \mid(t, x) \in \bigcap_{n}\left(C_{i \mid n} \times F_{i \mid n}\right)\right\}
$$

belongs to $(\mathcal{C} \times \mathscr{F}(X) \times \mathscr{F}(\mathscr{T}))_{\sigma \delta}$ and each set

$$
Q(t)=\{(x, i) \mid(t, x, i) \in Q\}
$$

is closed-valued because so are the $F_{i \mid n}$. We also have

$(\mathcal{C} \times \mathscr{F}(X) \times \mathscr{F}(\mathcal{K}))_{\sigma \delta} \subset(\mathcal{C} \times \mathscr{F}(X \times \mathscr{T}))_{o \delta} \subset \mathcal{Q}(\mathcal{C} \times \mathscr{F}(X \times \mathscr{F}))$

(since a countable union or intersection of Suslin sets is again a Suslin set).

It follows that for any closed $F \subset X \times \Re$,

$$
Q_{F}=Q \cap(T \times F) \in \mathbb{Q}(\mathcal{C} \times \mathscr{F}(X \times \Re)) .
$$

In view of Proposition 3, this implies that

$$
Q^{-}(F)=\operatorname{pr}_{T} Q_{F} \in \mathcal{Q}(\mathcal{C}) \text {. }
$$

In particular, $\operatorname{dom} M=\operatorname{dom} Q=\operatorname{pr}_{T} Q \in \mathbb{Q}(\mathcal{C})$. Therefore

$$
Q^{-}(U) \in(\mathbb{Q}(\mathcal{C}))_{\sigma} \subset \Sigma(\mathscr{Q}(\mathcal{C}))
$$

for any open $U \subset X \times \Re$.

By Theorem $1, Q$ is $\mathfrak{M}$-analytic. But $M(t)=\operatorname{pr}_{X} Q(t)$ and $\operatorname{pr}_{X}$ is a continuous mapping from $X \times \Re$ into $X$. Hence $M$ is also $\mathfrak{M}$-analytic.

Corollary 5.1. Let $X$ be a Suslin space (not necessarily metrizable), and let $T, \mathcal{C}$ and $\mathfrak{M}$ be the same as in the statement of Theorem 5. Assume that the graph of the set-valued mapping $M$ from $T$ into $X$ belongs to $\mathcal{Q}(\mathcal{C} \times \mathscr{B}(X))$. Then $M$ is $\mathfrak{M}$-analytic.

Proof. By the assumptions, $X$ is a continuous image of a Polish space $Y$. Let $h$ be the corresponding continuous mapping from $Y$ onto $X$. Consider the multifunction $S$ from $T$ into $Y$ defined by

$$
S(t)=h^{-1}(M(t)),
$$

so that $M(t)=h(S(t))$. Thus, to prove the corollary, we need to verify that $S$ is $\mathfrak{M}$-analytic. 
We have

$$
\operatorname{Gr} M=\bigcup_{i \in \mathscr{N}} \bigcap_{n}\left(C_{i \mid n} \times A_{i \mid n}\right)
$$

where $C_{i \mid n} \in \mathcal{C}$ and $A_{i \mid n}$ are either open or closed in $X$. (Indeed, $\mathscr{B}(X)$ is just the minimal family containing all open and closed subsets of $X$ and closed under countable unions and intersections. Thus the above formula follows from Corollary 2.1.) It follows that

$$
\operatorname{Gr} S=\bigcup_{i \in \Re} \bigcap_{n}\left(C_{i \mid n} \times h^{-1}\left(A_{i \mid n}\right)\right) \text {. }
$$

Since $h$ is a continuous mapping, the $h^{-1}\left(A_{i \mid n}\right)$ are either open or closed, hence belong to $\mathscr{B}(Y)$. Since $Y$ is Polish, Theorem 5 shows that $S$ is $\mathfrak{M}$-analytic.

Having examined the proofs of Corollary 5.1 and Theorem 5, one can easily observe that we have actually proved the following fact.

Corollary 5.2. Let $X, \mathcal{C}, \mathfrak{M}$ and $M$ be the same as in Corollary 5.1. Then

$$
M(t)=h(S(t))
$$

where $S$ is a closed-valued $\mathfrak{M}$-measurable multifunction from $T$ into a Polish space and $h$ is a continuous mapping from this Polish space into $X$.

This result is essentially due to Leese [9] who assumed, in addition, that $C$ is a $\sigma$-algebra and $\mathcal{C}=\mathscr{Q}(\mathcal{C})=\mathfrak{M}$. We shall call such families Suslin algebras. According to Leese, set-valued mappings which can be represented in the form (6) are called mappings of Suslin type. To be more specific, let us say that $M$ is of $\mathfrak{M}$-Suslin type if it is defined by (6) with $h$ continuous and $S$ closed-valued and $\mathfrak{M}$-measurable and having values in a Polish space. As is clear from the definitions and Theorem 1, any set-valued mapping of $\mathfrak{M}$-Suslin type is $\mathfrak{M}$-analytic. It is not so clear if the converse is true, though a weakened form of the converse can be easily derived from Theorems 4 and 5.

Corollary 5.3. Let $\mathfrak{M}$ be a $\sigma$-algebra of subsets of $T$, let $X$ be a Polish space, and let $M$ be an $\mathfrak{R}$-analytic multifunction from $T$ into $X$. Let $\mathfrak{R}_{1}=$ $\Sigma(\mathbb{Q}(\mathfrak{M}))$. Then $M$ is of $\mathfrak{R}_{1}-$ Suslin type.

Proof. According to Theorem 4, Gr $M \in \mathbb{Q}(\mathfrak{M} \otimes \mathscr{B}(X))$. Apply Corollary 5.2.

At the same time, all properties we are discussing here are equivalent in the case of Suslin algebras as seen from the following proposition.

Corollary 5.4. Let $X$ be a Suslin space, let $C$ be a family of subsets of $T$ containing $\varnothing$, let $\mathfrak{M}=\Sigma(\mathscr{Q}(\mathcal{C})$ ), and let $M$ be a set-valued mapping from $T$ into $X$. Then among the following three properties the implication (c) $\Rightarrow(b) \Rightarrow(a)$ is always valid with the full equivalence of the three if $X$ is a Polish space and 
$\mathcal{C}=\mathfrak{M}$ is a Suslin algebra:

(a) $M$ is $\mathfrak{M}$-analytic;

(b) $M$ is of $\mathfrak{M}$-Suslin type;

(c) $\operatorname{Gr} M \in \mathbb{Q}(\mathcal{C} \times \mathscr{B}(X))$.

Proof. Implication (c) $\Rightarrow$ (b) follows from Corollary $5.2,(b) \Rightarrow$ (a) is trivial, and if $X$ is a Polish space and $\mathcal{C}=\mathfrak{M}$ is a Suslin algebra, implication (a) $\Rightarrow$ (c) follows from Theorem 4 and Corollary 2.2 .

To conclude the paper, we consider several results concerning set-theoretical operations with analytic multifunctions.

Corollary 5.5. For multifunctions into Polish spaces and continuous mappings from one Polish space into another, the following are true:

(a) if $M$ is $\mathfrak{M}$-analytic, then the multifunction defined by $t \rightarrow h^{-1}(M(t))$ is $\mathfrak{M}_{1}$-analytic;

(b) if $M_{1}, M_{2}, \ldots$ are $\mathfrak{M}$-analytic, then $M=M_{1} \cap M_{2} \cap \ldots$ is $\mathfrak{M}_{1^{-}}$ analytic;

(c) if $M_{1}, M_{2}, \ldots$ are $\mathfrak{M}$-analytic, then $M=M_{1} \times M_{2} \times \ldots$ is $\mathfrak{M}_{1^{-}}$ analytic.

Here $\mathfrak{M}_{1}=\Sigma(\mathbb{Q}(\mathfrak{M}))$.

Proof. In each of the three cases the proof is straightforward from Theorems 4 and 5. In every case the graph of the multifunction in question belongs to the corresponding Suslin class by Theorem 4, and Theorem 5 yields the desired result.

For unions of analytic multifunctions, a stronger result is valid.

Proposition 6. Let $M_{1}, M_{2}, \ldots$ be a sequence of set-valued mappings from $T$ into a topological space $X$. If all $M_{k}$ are $\mathfrak{M}$-analytic and either $X$ is Polish or $\operatorname{dom} M_{k}=T$ for all $k=1,2, \ldots$, then the set-valued mapping

$$
t \rightarrow M(t)=\bigcup_{k=1}^{\infty} M_{k}(t)
$$

is also $\mathfrak{M}$-analytic.

Proof. Let $\left(Z_{k}, f_{k}\right)$ represent $M_{k}, k=1,2, \ldots, Z_{k}$ being a Polish space and $f_{k}: T \times Z_{k} \rightarrow X$ being $\mathfrak{M}$-Carathéodory. Let $Z$ be the topological direct sum of the $Z_{k}$, which is to say, $Z=Z_{1} \cup Z_{2} \cup \ldots$ (the $Z_{k}$ are considered as disjoint sets) with the base of topology formed by all finite unions $U_{k_{1}}$ $\cup \cdots \cup U_{k_{s}}$, where each $U_{k_{r}}$ belongs to a fixed countable base of $Z_{k_{r}}$.

The formula

$$
f(t, z)=f_{k}(t, z), \quad \text { if } z \in Z_{k},
$$

defines a mapping from $T \times Z$ into $X$ which is obviously $\mathfrak{M}$-Carathéodory. If $\operatorname{dom} M_{k}=T$ for all $k$, then $f(t, Z)=\cup_{k} f\left(t, Z_{k}\right)=M(t)$ for all $t$ which 
shows that $M$ is actually represented by $(Z, f)$.

If the sets dom $M_{k}$ are different for different $k$, we consider the following multifunction $S$ from $T$ into $Z$ :

$$
S(t)=\bigcup_{k \in K(t)} Z_{k},
$$

where $K(t)=\left\{k \in N \mid t \in \operatorname{dom} M_{k}\right\}$. Then $S$ is closed-valued and $\mathbb{M}$-measurable because

$$
\left\{t \mid S(t) \cap Z_{k} \neq \varnothing\right\}=\operatorname{dom} M_{k}
$$

and hence $\mathfrak{M}$-analytic by Theorem 1 .

Let $(V, g)$ represent $S$. Then setting $h(t, v)=f(t, g(t, v))$, we see that $(V, h)$ represents $M$. Indeed, if $t \in \operatorname{dom} M, x \in M(t)$, then there is an integer $k$ such that $x \in M_{k}(t)$, hence there is $z_{k} \in Z_{k}$ such that $f\left(t, z_{k}\right)=x$. Therefore $z_{k} \in S(t)$ and there is $v \in V$ such that $g(t, v)=z_{k}$. Obviously $h$ is continuous in $v$. It is also $\mathfrak{M}$-measurable in $t$ if $X$ is Polish since in this case the function $t \rightarrow f(t, z(t))$ is $\mathfrak{M}$-measurable whenever $z(t)$ is $\mathfrak{M}$-measurable.

\section{REFERENCES}

1. R. J. Aumann, Measurable utility and measurable choice theorem, La Décision (Actes Colloq. Internat. du Centre Nat. Recherche Sci., Aix-en-Provence, 1967), Editions du CRNS, Paris, 1969, pp. 15-26.

2. D. W. Bressler and M. Sion, The current theory of analytic sets, Canad. J. Math. 16 (1964), 207-230.

3. C. Castaing, Multi-applications mesurables, Rev. Informat. Recherche Opérationelle 1 (1967), 91-126.

4. C. Dellacherie et P.-A. Meyer, Probabilités et potentiel, Hermann, Paris, 1975.

5. J. Hoffmann-Jorgensen, The theory of analytic spaces, Various Publ. Ser. no. 10, Aarhus Univ., Denmark, 1970.

6. A. D. Ioffe, Representation theorems for multifunctions and analytic sets, Bull. Amer. Math. Soc. 84 (1978), 142-144.

7. K. Kuratowski, Topology, Vol. 1, Academic Press, New York, 1966.

8. K. Kuratowski and C. Ryll-Nardzewski, A general theorem on selectors, Bull. Acad. Polon. Sci. Sér. Sci. Math. Astronom. Phys. 13 (1965), 397-403.

9. S. J. Leese, Multifunctions of Suslin type, Bull. Austral. Math. Soc. 11 (1974), 395-411.

10. __ Measurable selections and uniformization of Suslin sets, Amer. J. Math. (to appear).

11. V. L. Levin, Measurable selections of set-valued mappings and projections of measurable sets, Funkcional. Anal. i Priložen. (to appear).

12. P. S. Novikov, On projections of certain B-sets, Dokl. Akad. Nauk SSSR 23 (1939), 863-866.

13. V. A. Rokhlin, Selected topics from the metric theory of dynamical systems, Amer. Math. Soc. Transl. 49 (1966), 171-240.

14. J. Saint-Pierre, Caractérisation des $\mathcal{F}$-sousliniens d'un produit et théorèmes de sections, Travaux du Sém. d'Analyse Convexe, exp. 8, vol. 7, 1977.

15. S. Simons, A proof that Souslin Souslin H C Souslin H, Canad. Math. Bull. 9 (1966), 79-82.

Profsojuznaja 97-1-203, Moscow B-279, U.S.S.R. 